Janusz Matkowski (Zielona Góra and Katowice) ŁukAsz StetTner (Warszawa)

\title{
ON THE BELLMAN EQUATION FOR ASYMPTOTICS OF UTILITY FROM TERMINAL WEALTH
}

Abstract. The asymptotics of utility from terminal wealth is studied. First, a finite horizon problem for any utility function is considered. To study a long run infinite horizon problem, a certain positive homogeneity $(\mathrm{PH})$ assumption is imposed. It is then shown that assumption $(\mathrm{PH})$ is practically satisfied only by power and logarithmic utility functions.

1. Finite horizon problem. Consider a market with $d$ assets, the prices of which are modelled in discrete time by the formula

$$
\frac{S_{i}(n+1)}{S_{i}(n)}=\zeta_{i}(z(n), \xi(n)):=\zeta_{i}(n),
$$

where $S_{i}(n)$ is the price of the $i$ th asset at time $n,(z(n))$ is a Markov process of economic factors taking values in a measurable space $D$, and $(\xi(n))$ is a sequence of i.i.d. random variables. We denote by $\zeta(n)$ the vector of ratios $S_{i}(n+1) / S_{i}(n)$. Starting with an initial wealth $W$ we invest in assets, denoting by $\pi_{i}^{n}$ the portion of capital invested at time $n$ in the $i$ th asset. It is clear that the vector $\pi^{n}$ consisting of the $\pi_{i}^{n}$ for $i=1, \ldots, d$ is our investment strategy at time $n$ and $\pi^{n} \in \mathcal{S}=\left\{\eta \in \mathbb{R}^{d}: \eta_{i} \geq 0, \sum_{i=1}^{d} \eta_{i}=1\right\}$. The wealth process $W(n)$ at time $n$ is then given by the formula

$$
W(n)=W(n-1)\left(\pi^{n-1} \cdot \zeta(n-1)\right)=W(0) \prod_{i=0}^{n-1}\left(\pi^{i} \cdot \zeta(i)\right)
$$

with - standing for the scalar product. Consider now an increasing concave continuous function $U:(0, \infty) \rightarrow \mathbb{R}$ which is called a utility function. We

2010 Mathematics Subject Classification: Primary 91G10; Secondary 93E20.

Key words and phrases: asymptotics, optimal portfolio, utility, terminal wealth, Bellman equation. 
study first the following problem. Given a fixed time horizon $T$, initial wealth $W(0)=W$ and initial value of the process of economic factors $z(0)=z$, find an investment strategy $V=\left(\pi^{0}, \pi^{1}, \ldots, \pi^{T-1}\right)$ for which the value of $E_{W, z}\{U(W(T))\}$ is maximal, where by $E_{W, z}$ we denote the conditional expectation given initial values $W$ and $z$ of the wealth and economic factors respectively. Let a measurable function $\nu^{T}:(0, \infty) \times D \rightarrow \mathbb{R}$ be such that

$$
U\left(\nu^{T}(W, z)\right)=\sup _{V} E_{W, z}\{U(W(T))\} .
$$

We would like to characterize the function $\nu^{T}$ and to find a recursive formula for optimal investment strategies. For this purpose we introduce the following system of Bellman equations:

$$
\begin{aligned}
\nu_{T}^{T}(W, z) & :=W, \\
\nu_{T-1}^{T}(W, z) & :=\sup _{\pi^{T-1} \in \mathcal{S}} U^{-1}\left(E_{z}\left\{U\left(\nu_{T}^{T}\left(W\left(\pi^{T-1} \cdot \zeta(T-1)\right), z(1)\right)\right)\right\}\right), \\
\nu_{T-2}^{T}(W, z) & :=\sup _{\pi^{T-2} \in \mathcal{S}} U^{-1}\left(E_{z}\left\{U\left(\nu_{T-1}^{T}\left(W\left(\pi^{T-2} \cdot \zeta(T-2)\right), z(1)\right)\right)\right\}\right), \\
& \cdots \\
\nu_{0}^{T}(W, z) & :=\sup _{\pi^{0} \in \mathcal{S}} U^{-1}\left(E_{z}\left\{U\left(\nu_{1}^{T}\left(W\left(\pi^{0} \cdot \zeta(0)\right), z(1)\right)\right)\right\}\right),
\end{aligned}
$$

where by $E_{z}$ we denote the conditional expectation given initial value $z$ of the process $(z(n))$.

THEOREM 1.1. We have

$$
\begin{aligned}
& \nu_{0}^{T}(W, z)=\sup _{\pi^{0} \in \mathcal{S}} U^{-1}\left(E _ { z } \left\{\operatorname { s u p } _ { \pi ^ { 1 } \in \mathcal { S } } E _ { z ( 1 ) } \left\{\operatorname { s u p } _ { \pi ^ { 2 } \in \mathcal { S } } E _ { z ( 1 ) } \left\{\ldots \sup _{\pi^{T-1} \in \mathcal{S}}\right.\right.\right.\right. \\
&\left.\left.\left.\left.E_{z(1)}\left\{U\left(W\left(\pi^{T-1} \cdot \zeta(T-1)\right) \ldots\left(\pi^{0} \cdot \zeta(0)\right)\right)\right\}\right\}\right\}\right\}\right)=\nu^{T}(W, z) .
\end{aligned}
$$

Let $\hat{\pi}^{t}:(0, \infty) \times D \rightarrow \mathcal{S}$ be a selector, i.e. a Borel measurable function for which the supremum in

$$
\nu_{t}^{T}(W, z)=\sup _{\pi^{t} \in \mathcal{S}} U^{-1}\left(E_{z}\left\{U\left(\nu_{t+1}^{T}\left(W\left(\pi^{t} \cdot \zeta(t)\right), z(1)\right)\right)\right\}\right)
$$

is attained. Then the control

$$
\hat{V}=\left(\hat{\pi}^{0}(W, z), \hat{\pi}^{1}(W(1), z(1)), \ldots, \hat{\pi}^{T-1}(W(T-1), z(T-1))\right)
$$

is optimal for $(1.3)$.

Proof. Notice first that since $U^{-1}$ is increasing we are allowed to change the order of the sup and $U^{-1}$. Therefore substituting the value of $\nu_{T-1}^{T}$ in 
the definition of $\nu_{T-2}^{T}$ we obtain

$$
\begin{aligned}
\nu_{T-2}^{T}(W, z)= & \sup _{\pi^{T-2} \in \mathcal{S}} U^{-1}\left(E _ { z } \left\{U \left(\sup _{\pi^{T-1} \in \mathcal{S}}\right.\right.\right. \\
& \left.\left.\left.U^{-1}\left(E_{z(1)}\left\{U\left(W\left(\pi^{T-1} \cdot \zeta(T-1)\right)\left(\pi^{T-2} \cdot \zeta(T-2)\right)\right)\right\}\right)\right)\right\}\right) \\
& \sup _{\pi^{T-2} \in \mathcal{S}} U^{-1}\left(E _ { z } \left\{\sup _{\pi^{T-1} \in \mathcal{S}}\right.\right. \\
& \left.\left.\left.E_{z(1)}\left\{U\left(W\left(\pi^{T-1} \cdot \zeta(T-1)\right)\left(\pi^{T-2} \cdot \zeta(T-2)\right)\right)\right\}\right)\right\}\right) .
\end{aligned}
$$

Hence by backward induction we obtain the first part of the formula (1.5). Using standard arguments (see [3] or [8]) we see that $\nu_{0}^{T}=\nu^{T}$ and that the control $\hat{V}$ is optimal.

The following assumption will be important:

(PH) for a bounded positive random variable $X$ the mapping

$$
t \mapsto U^{-1} E_{z}\{U(t X)\}
$$

is positively homogeneous, i.e. for $t>0$ we have

$$
U^{-1} E_{z}\{U(t X)\}=t U^{-1} E_{z}\{U(X)\} .
$$

Consider now the following system of Bellman equations:

$$
\begin{aligned}
\bar{\nu}_{T-1}^{T}(z) & :=\sup _{\pi^{T-1} \in \mathcal{S}} U^{-1}\left(E_{z}\left\{U\left(\left(\pi^{T-1} \cdot \zeta(T-1)\right)\right)\right\}\right) \\
\bar{\nu}_{T-2}^{T}(z) & :=\sup _{\pi^{T-2} \in \mathcal{S}} U^{-1}\left(E_{z}\left\{U\left(\left(\pi^{T-2} \cdot \zeta(T-2)\right) \bar{\nu}_{T-1}^{T}(z(1))\right)\right\}\right) \\
& \cdots \\
\bar{\nu}_{0}^{T}(z) & :=\sup _{\pi^{0} \in \mathcal{S}} U^{-1}\left(E_{z}\left\{U\left(\left(\pi^{0} \cdot \zeta(0)\right) \bar{\nu}_{1}^{T}(z(1))\right)\right\}\right) .
\end{aligned}
$$

Proposition 1.2. Under $(\mathrm{PH})$ we have $W \bar{\nu}_{0}^{T}(z)=\nu^{T}(W, z)$, which means that optimal expected utility from terminal wealth is positively homogeneous with respect to the initial value of the wealth. Moreover, the control $\bar{V}=\left(\bar{\pi}^{0}(z), \bar{\pi}^{1}(z(1)), \ldots, \bar{\pi}^{T-1}(z(T-1))\right)$, where $\bar{\pi}^{t}: D \rightarrow \mathcal{S}$ is a selector i.e. a Borel measurable function for which the supremum in

$$
\bar{\nu}_{t}^{T}(z):=\sup _{\pi^{t} \in \mathcal{S}} U^{-1}\left(E_{z}\left\{U\left(\left(\pi^{t} \cdot \zeta(t)\right) \bar{\nu}_{t+1}^{T}(z(1))\right)\right\}\right)
$$

is attained, is optimal.

Proof. Using (PH) we have

$$
\begin{aligned}
\bar{\nu}_{T-2}^{T}(z)= & \sup _{\pi^{T-2} \in \mathcal{S}} U^{-1}\left(E _ { z } \left\{\sup _{\pi^{T-1} \in \mathcal{S}}\right.\right. \\
& \left.\left.\quad\left(E_{z(1)}\left\{U\left(\left(\pi^{T-2} \cdot \zeta(T-2)\right)\left(\pi^{T-1} \cdot \zeta(T-1)\right) \bar{\nu}_{T-1}^{T}(z(1))\right)\right\}\right)\right\}\right)
\end{aligned}
$$


whence, as in the proof of Theorem 1.1, we obtain

$$
\begin{aligned}
\bar{\nu}_{0}^{T}(z)=\sup _{\pi^{0} \in \mathcal{S}} U^{-1}\left(E _ { z } \left\{\operatorname { s u p } _ { \pi ^ { 1 } \in \mathcal { S } } E _ { z ( 1 ) } \left\{\operatorname { s u p } _ { \pi ^ { 2 } \in \mathcal { S } } E _ { z ( 1 ) } \left\{\ldots \sup _{\pi^{T-1} \in \mathcal{S}}\right.\right.\right.\right. \\
\left.\left.\left.\left.E_{z(1)}\left\{U\left(\left(\pi^{T-1} \cdot \zeta(T-1)\right) \ldots\left(\pi^{0} \cdot \zeta(0)\right)\right)\right\}\right\}\right\}\right\}\right)
\end{aligned}
$$

and by $(\mathrm{PH})$ again it coincides with $(1 / W) \nu^{T}(W, z)$. The form of the optimal strategy $\bar{V}$ follows from [3] or [8].

We then see that the problem of finite horizon utility from terminal wealth can be solved for any utility function, but under assumption $(\mathrm{PH})$ the problem can be simplified. A natural question is to evaluate optimal asymptotics, i.e. to find $\lambda$ such that $U\left(e^{\lambda n}\right) \sim \sup _{V} E\{U(W(n))\}$ for sufficiently large $n$. This problem leads to a suitable infinite horizon Bellman equation which has been studied in particular in [6] and [7], or [5] (see also references therein) for power and logarithmic utility functions. The problem is to find a unified general approach for any utility function. We formulate below such a Bellman equation. From its form a certain positive homogeneity condition (satisfied under (PH)) should be satisfied. As we show below in Section 3, condition $(\mathrm{PH})$ only holds for power or logarithmic utility functions, possibly shifted by a constant.

2. Infinite horizon asymptotics Bellman equation. Consider an infinite horizon problem. We want to find a continuous bounded function $\nu:(0, \infty) \times D \rightarrow \mathbb{R}$ and a constant $\lambda$ such that for $W \in(0, \infty), z \in D$ and $K \in(0, \infty)$,

$$
K e^{\nu(W, z)}=\sup _{\pi \in \mathcal{S}} U^{-1}\left(E_{z}\left\{U\left((\pi \cdot \zeta(0)) e^{-\lambda} K e^{\nu(W(\pi \cdot \zeta(0)), z(1))}\right)\right\}\right) .
$$

The form of this Bellman equation is justified by the following

THEOREM 2.1. If there is a continuous bounded function $\nu:(0, \infty) \times$ $D \rightarrow \mathbb{R}$ and a constant $\lambda$ such that for any $W \in(0, \infty), z \in D$ and $K \in$ $(0, \infty)$ equation 2.1 is satisfied then

$$
\lambda=\sup _{V} \liminf _{T \rightarrow \infty} \frac{1}{T} \ln U^{-1}\left(E_{z}\{U(W(T))\}\right) .
$$

Furthermore, if $\tilde{\pi}:(0, \infty) \times D \rightarrow \mathcal{S}$ is a selector of the right hand side of (2.1), then the control $\tilde{V}=(\tilde{\pi}(W(i), z(i)))$ is optimal, i.e. for this control the value of $\liminf _{T \rightarrow \infty} T^{-1} \ln U^{-1}\left(E_{z}\{U(W(T))\}\right)$ is equal to $\lambda$.

Proof. Notice first that by (2.1) the mapping

$$
K \mapsto \sup _{\pi \in \mathcal{S}} U^{-1}\left(E_{z}\left\{U\left((\pi \cdot \zeta(0)) e^{-\lambda} K e^{\nu(W(\pi \cdot \zeta(0)), z(1))}\right)\right\}\right)
$$


is positively homogeneous. Therefore iterating (2.1) we have

$$
\begin{aligned}
e^{\nu(W, z)}= & \sup _{\pi \in \mathcal{S}} U^{-1}\left(E _ { z } \left\{U \left((\pi \cdot \zeta(0)) e^{-\lambda} \sup _{\pi^{1} \in \mathcal{S}}\right.\right.\right. \\
& \left.\left.U^{-1}\left(E_{z(1)}\left\{U\left(\left(\pi^{1} \cdot \zeta(1)\right) e^{-\lambda} e^{\nu\left(W(\pi \cdot \zeta(0))\left(\pi^{1} \cdot \zeta(1)\right), z(1)\right)}\right)\right\}\right)\right\}\right) \\
= & e^{-2 \lambda} \sup _{\pi \in \mathcal{S}} U^{-1}\left(E _ { z } \left\{\sup _{\pi^{1} \in \mathcal{S}}\right.\right. \\
& \left.\left(E_{z(1)}\left\{U\left((\pi \cdot \zeta(0))\left(\pi^{1} \cdot \zeta(1)\right) e^{\left.\nu\left(W(\pi \cdot \zeta(0))\left(\pi^{1} \cdot \zeta(1)\right), z(1)\right)\right)}\right\}\right)\right\}\right)
\end{aligned}
$$

and, by induction, we obtain

$$
\begin{gathered}
e^{\nu(W, z)}=e^{-n \lambda} \sup _{\pi \in \mathcal{S}} U^{-1}\left(E _ { z } \left\{\operatorname { s u p } _ { \pi ^ { 1 } \in \mathcal { S } } E _ { z ( 1 ) } \left\{\ldots \sup _{\pi^{n-1} \in \mathcal{S}}\right.\right.\right. \\
\left.\left.\left.E_{z(1)}\left\{U\left((\pi \cdot \zeta(0)) \prod_{i=1}^{n-1}\left(\pi^{i} \cdot \zeta(i)\right) e^{\nu\left(W(\pi \cdot \zeta(0)) \prod_{i=1}^{n-1}\left(\pi^{i} \cdot \zeta(i)\right), z(1)\right)}\right)\right\}\right\}\right\}\right) .
\end{gathered}
$$

By positive homogeneity again we have

$$
\begin{aligned}
W e^{\nu(W, z)}=e^{-n \lambda} \sup _{\pi \in \mathcal{S}} U^{-1}\left(E _ { z } \left\{\operatorname { s u p } _ { \pi ^ { 1 } \in \mathcal { S } } E _ { z ( 1 ) } \left\{\ldots \sup _{\pi^{n-1} \in \mathcal{S}}\right.\right.\right. \\
\left.\left.\left.E_{z(1)}\left\{U\left(W(n) e^{\nu(W(n), z(1))}\right)\right\}\right\}\right\}\right)
\end{aligned}
$$

and

$$
\begin{gathered}
W e^{\nu(W, z) \pm\|\nu\|}=e^{-n \lambda} \sup _{\pi \in \mathcal{S}} U^{-1}\left(E _ { z } \left\{\operatorname { s u p } _ { \pi ^ { 1 } \in \mathcal { S } } E _ { z ( 1 ) } \left\{\ldots \sup _{\pi^{n-1} \in \mathcal{S}}\right.\right.\right. \\
\left.\left.\left.E_{z(1)}\left\{U\left(W(n) e^{\nu(W(n), z(1)) \pm\|\nu\|}\right)\right\}\right\}\right\}\right),
\end{gathered}
$$

where $\|\nu\|=\sup _{W \in(0, \infty), z \in D}|\nu(W, z)|$. Consequently,

$$
\begin{aligned}
e^{n \lambda} W e^{\nu(W, z)-\|\nu\|} \leq \sup _{\pi \in \mathcal{S}} U^{-1}\left(E _ { z } \left\{\operatorname { s u p } _ { \pi ^ { 1 } \in \mathcal { S } } E _ { z ( 1 ) } \left\{\ldots \sup _{\pi^{n-1} \in \mathcal{S}}\right.\right.\right. \\
\left.\left.\left.E_{z(1)}\{U(W(n))\}\right\}\right\}\right) \leq e^{n \lambda} W e^{\nu(W, z)+\|\nu\|} .
\end{aligned}
$$

Taking the logarithm in $(2.6)$, dividing by $n$ and letting $n \rightarrow \infty$ we obtain (2.2). The optimality of $\tilde{V}$ follows from standard arguments (see [3] or [8]).

Notice that under $(\mathrm{PH})$ the equation (2.1) can be written in the form

$$
e^{\nu(W, z)+\lambda}=\sup _{\pi \in \mathcal{S}} U^{-1}\left(E_{z}\left\{U\left((\pi \cdot \zeta(0)) e^{\nu(W(\pi \cdot \zeta(0)), z(1))}\right)\right\}\right)
$$

and $\nu$, as in Proposition 1.1, may not depend on $W$. Consequently, we may look for a solution to the equation

$$
e^{\nu(z)+\lambda}=\sup _{\pi \in \mathcal{S}} U^{-1}\left(E_{z}\left\{U\left((\pi \cdot \zeta(0)) e^{\nu(z(1))}\right)\right\}\right) .
$$

By similar considerations to the proof of Theorem 2.1 we have 
COROLlary 2.2. If there exists a continuous bounded function $\nu: D \rightarrow$ $\mathbb{R}$ and a constant $\lambda$ for which the equation (2.7) is satisfied then

$$
\lambda=\sup _{V} \ln U^{-1}(E\{U(W(T))\})
$$

and an optimal strategy is $\tilde{V}=(\tilde{\pi}(z(i)))$, where $\tilde{\pi}$ is a selector for which the supremum on the right hand side of (2.7) is attained.

REMARK 2.3. If the random rate of return (1.1) does not depend on the process of economic factors $(z(n))$, then the optimal strategy is stationary, i.e. we choose the same portfolio strategy $\pi$ at each time and it coincides with the finite horizon optimal strategy given by a suitable version of Proposition 1.2. If we consider proportional transaction costs then as in [5] (see also [6] and [7]) the solutions to Bellman equations depend on the current value of the process $\pi$ before a possible transaction. In Theorem 2.1 and Corollary 2.2 we assume the existence of a bounded solution to the Bellman equations (2.1) or 2.7). Sufficient conditions for the existence of solutions to Bellman equations in particular cases of power and logarithmic utilities are formulated in [5]-[7].

3. Characterization of the class of utility functions satisfying condition $(\mathbf{P H})$. We first consider the case of condition $(\mathrm{PH})$ within the class of binomial random variables $X$ taking one value, say $x$, with a fixed probability $a$ and a second value, say $y$, with probability $1-a$.

Theorem 3.1. Let $a \in(0,1)$ be fixed. Suppose that $U:(0, \infty) \rightarrow \mathbb{R}$ is a continuous and strictly monotonic function. Then

$$
U^{-1}(a U(t x)+(1-a) U(t y))=t U^{-1}(a U(x)+(1-a) U(y))
$$

for all $t, x, y>0$ if and only if there are $A, B \in \mathbb{R}, A \neq 0$, such that either

$$
U(t)=A t^{p}+B, \quad t>0,
$$

or

$$
U(t)=A \log t+B, \quad t>0 .
$$

Proof. By the assumption on $U$ the set $J:=U((0, \infty))$ is an open interval. For any $u, v \in J$ there are unique $x, y \in(0, \infty)$ such that $x=U^{-1}(u)$, $y=U^{-1}(v)$. Substituting these values in (3.1) and taking the value $U$ of both sides we obtain

$$
\left(U \circ t U^{-1}\right)(a u+(1-a) v)=a\left(U \circ t U^{-1}\right)(u)+(1-a)\left(U \circ t U^{-1}\right)(v)
$$

for $t>0, u, v \in J$, which means that, for any fixed $t>0$, the function $\gamma:=U \circ t U^{-1}$ satisfies the equation

$$
\gamma(a u+(1-a) v)=a \gamma(u)+(1-a) \gamma(v)
$$


for $u, v \in J$. Applying the Dároczy-Páles identity (see [2])

$$
\frac{u+v}{2}=a\left(a \frac{u+v}{2}+(1-a) v\right)+(1-a)\left(a u+(1-a) \frac{u+v}{2}\right)
$$

we hence get

$$
\begin{aligned}
\gamma\left(\frac{u+v}{2}\right)= & \gamma\left(a\left(a \frac{u+v}{2}+(1-a) v\right)\right. \\
& \left.+(1-a)\left(a u+(1-a) \frac{u+v}{2}\right)\right) \\
= & a \gamma\left(a \frac{u+v}{2}+(1-a) v\right)+(1-a) \gamma\left(a u+(1-a) \frac{u+v}{2}\right) \\
= & a^{2} \gamma\left(\frac{u+v}{2}\right)+a(1-a)[\gamma(v)+\gamma(u)]+(1-a)^{2} \gamma\left(\frac{u+v}{2}\right),
\end{aligned}
$$

whence

$$
\gamma\left(\frac{u+v}{2}\right)=\frac{\gamma(u)+\gamma(v)}{2}, \quad u, v \in J
$$

that is, $\gamma=U \circ t U^{-1}$ is Jensen affine in $J$. The continuity of $U \circ t U^{-1}$ implies that, for any $t>0$, it is an affine function in $J$ (see [4]). Consequently, for any $t>0$, there are unique $m(t), k(t) \in \mathbb{R}$ such that

$$
\left(U \circ t U^{-1}\right)(u)=m(t) u+k(t), \quad t>0, u \in J .
$$

The continuity of $U \circ t U^{-1}$ implies that the functions $m, k:(0, \infty) \rightarrow \mathbb{R}$ are continuous. Since $U \circ t U^{-1}$ is strictly increasing, we hence get

$$
m(t)>0, \quad t>0 .
$$

From (3.2), for arbitrary $s, t>0$ and $u \in J$ we have

$$
\begin{aligned}
\left(U \circ s t U^{-1}\right)(u) & =\left[\left(U \circ s U^{-1}\right) \circ\left(U \circ t U^{-1}\right)\right](u) \\
& =m(s)\left[\left(U \circ t U^{-1}\right) u\right]+k(s) \\
& =m(s) m(t) u+m(s) k(t)+k(s),
\end{aligned}
$$

and obviously, by (3.2),

$$
\left(U \circ s t U^{-1}\right)(u)=m(s t) u+k(s t), \quad s, t>0, u \in J .
$$

Both these equations imply that

$$
m(s t)=m(s) m(t), \quad s, t>0,
$$

and

$$
k(s t)=m(s) k(t)+k(s), \quad s, t>0 .
$$

The continuity of $U$, 3.3) and Corollary of Theorem 14.4 from [1] imply that

$$
m(t)=t^{p}, \quad t>0,
$$

for some $p \in \mathbb{R}$. 
Consider first the case when $p \neq 0$. From (3.4) we get

$$
k(s t)=s^{p} k(t)+k(s), \quad s, t>0 .
$$

Hence, by symmetry of the right-hand side,

$$
s^{p} k(t)+k(s)=t^{p} k(s)+k(t), \quad s, t>0,
$$

we obtain

$$
\frac{k(t)}{t^{p}-1}=\frac{k(s)}{s^{p}-1}, \quad s, t>0,
$$

which, together with the continuity of $k$, implies that, for some $c \in \mathbb{R}$,

$$
k(t)=c\left(t^{p}-1\right), \quad t>0 .
$$

From (3.2), (3.5) and (3.6) we get

$$
\left(U \circ t U^{-1}\right)(u)=t^{p} u+c\left(t^{p}-1\right), \quad t>0, u \in J .
$$

Setting here $u=U(1)$ we get

$$
U(t)=A t^{p}+B, \quad t>0,
$$

for some $A, B \in \mathbb{R}, A \neq 0$.

In the remaining case, when $p=0$, in view of (3.5),

$$
m(t)=1, \quad t>0 .
$$

Hence, taking into account (3.4) and Theorem 14.4 from [1] we get

$$
k(s t)=k(t)+k(s), \quad s, t>0,
$$

whence, by the continuity of $k$, we obtain

$$
k(t)=A \log t, \quad t>0,
$$

for some $A \in \mathbb{R}$. Now from (3.2), (3.7) and 3.8$)$ we obtain

$$
\left(U \circ t U^{-1}\right)(u)=u+A \log t, \quad t>0, u \in J .
$$

As $U \circ t U^{-1}$ is increasing, this implies that $A>0$. Taking here $u=U(1)$ we obtain

$$
U(t)=A \log t+B, \quad t>0,
$$

for some $B \in \mathbb{R}$. This completes the proof.

We have just shown that condition $(\mathrm{PH})$ is satisfied for specific binomial random variables if utility functions are shifted by a constant power or logarithmic utilities. Almost immediately we therefore obtain the following equivalence

Corollary 3.2. Let $(\Omega, \Sigma, P)$ be a probability space such that there is an $A \in \Sigma$ with $\mu(A) \in(0,1)$, and let $U:(0, \infty) \rightarrow \mathbb{R}$ be a continuous and strictly monotonic function. The following two conditions are equivalent:

1. for any random variable $X: \Omega \rightarrow(0, \infty)$,

$$
U^{-1}(E\{U(t X)\})=t U^{-1}(E\{U(X)\}) \quad t>0,
$$


2. there are $A, B \in \mathbb{R}, A \neq 0$, such that either

$$
U(t)=A t^{p}+B, \quad t>0, \quad \text { or } \quad U(t)=A \log t+B, \quad t>0 .
$$

Proof. To prove that condition 1 implies condition 2 , for any $x, y>0$ take the random variable $X=x I_{A}+y I_{\Omega \backslash A}$. with $P(A)=a \in(0,1)$. Condition 1 then gives

$$
U^{-1}(a U(t x)+(1-a) U(t y))=t U^{-1}(a U(x)+(1-a) U(y)), \quad t, x, y>0,
$$

that is, equation (3.1). By Theorem 3.1 we obtain the desired implication. Since the reverse implication is obvious, the proof is complete.

Acknowledgements. The research of the second author was supported by MNiSzW grant no. N N201 371836. The authors would like to thank Dr. Jan Palczewski for helpful comments and suggestions.

\section{References}

[1] P. Billingsley, Probability and Measure, Wiley, 1979,

[2] Z. Dároczy and Z. Páles, Convexity with given infinite weight sequences, Stochastica 11 (1987), 5-12.

[3] O. Hernández-Lerma and J. B. Lasserre, Discrete-Time Markov Control Processes, Springer, 1996,

[4] M. Kuczma, An Introduction to the Theory of Functional Equations and Inequalities. Cauchy's Equation and Jensen's Inequality, PWN, Warszawa-Katowice, 1985.

[5] Ł. Stettner, Asymptotics of HARA utility from terminal wealth under proportional transaction costs with decision lag or execution delay and obligatory diversification, submitted.

[6] - Discrete time risk sensitive portfolio optimization with consumption and proportional transaction costs, Appl. Math. (Warsaw) 32 (2005),395-404.

[7] - Long time growth optimal portfolio with transaction costs, in: Banach Center Publ. 83, Inst. Math., Polish Acad. Sci., 2008, 231-241.

[8] J. Zabczyk, Chance and Decision. Stochastic Control in Discrete Time, Quaderni, Scuola Normale Superiore, Pisa, 1996.

Janusz Matkowski

Institute of Mathematics,

Computer Science and Econometrics

University of Zielona Góra

Podgórna 50

65-246 Zielona Góra, Poland

E-mail: J.Matkowski@wmie.uz.zgora.pl

and

Institute of Mathematics

Silesian University

40-007 Katowice, Poland
Łukasz Stettner

Institute of Mathematics Polish Academy of Sciences Śniadeckich 8 00-956 Warszawa, Poland and

Academy of Finance E-mail: stettner@impan.pl

Received on 1.10.2009; 
